\title{
On some robust algorithms for the Robin inverse problem
}

\author{
Mohamed Jaoua a - Slim Chaabane ${ }^{b}$ - Chokri Elhechmi b - Juliette \\ Leblond ${ }^{\mathrm{c}}$ - Moncef Mahjoub ${ }^{a}{ }^{*}$ — Jonathan R. Partington ${ }^{\mathrm{d}}$
}

a Laboratoire J.-A. Dieudonné, Université de Nice Sophia Antipolis, Parc Valrose, F-06108 Nice Cedex 02, FRANCE jaoua@math.unice.fr — ${ }^{*}$ Corresponding author : moncef.mahjoub@math.unice.fr

b LAMSIN-ENIT, BP 37, 1002 Tunis Belvédère, TUNISIA,

slim.chaabane@fsm.rnu.tn, chokri.elhechmi@enit.rnu.tn

c INRIA, BP 93, 06902 Sophia-Antipolis Cedex, FRANCE, leblond@sophia.inria.fr

d School of Mathematics, University of Leeds, Leeds LS2 9JT, U.K.,

J.R.Partington@leeds.ac.uk

\begin{abstract}
|
ABSTRACT. The problem we are dealing with is to recover a Robin coefficient (or impedance) from measurements performed on some part of the boundary of a domain, in the framework of nondestructive testing by the means of Electric Impedance Tomography. The impedance can provide information on the location of a corroded area, as well as on the extent of the damage, which has possibly occurred on an unaccessible part of the boundary. Two different identification algorithms are presented and studied: the first one is based on a Kohn and Vogelius cost function, actually an energetic least squares one, which turns the inverse problem into an optimization one ; as for the second, it makes use of the best approximation in Hardy classes, in order to extend the Cauchy data to the unreachable part of the boundary, and then compute the Robin coefficient from these extended data. Special focus is put on the robustness with respect to noise, both from a mathematical and and numerical point of wiew. Some numerical experiments are eventually presented and compared.
\end{abstract}

RÉSUMÉ. Dans ce travail nous nous sommes intéressé à un problème d'identification d'un coefficient de Robin (ou une impédence) à partir de mesures effectuées sur une certaine partie de la frontière d'un domaine. Ce problème est motivé par le contrôle non destructif des matériaux en tomographie par impédance électrique. L'impédance peut fournir des informations sur l'emplacement d'une zone de corrosion, ainsi que sur l'étendue des dommages, qui a peut-être eu lieu sur une partie inaccessible de la frontière. Deux algorithmes d'identification sont présentés et étudiés: le premier est basé sur la minimisation des fonctionnelles d'écart énérgitiques, dite de Kohn et Vogelius, comme pour le second, il fait usage à l'approximation dans les classes de Hardy afin de prolonger les données de Cauchy à la partie inaccessible de la frontière, puis calculer le coefficient de Robin qui est le quotient de ces données étendues. L'accent est mis sur la robustesse par rapport au bruit, à la fois d'un point de vu mathématique et numérique. Des expériences numériques sont finalement présentées et comparées.

KEYWORDS : Inverse problems; Cauchy problem; Robin coefficient; Stability; Hardy classes; Recovery algorithms

MOTS-CLÉS : Problèmes inverses; Problème de Cauchy; Coefficient de Robin; Stabilité; Algorithmes d'identification

|IIIIIIIIIIIIIIIIIIIIIIIIIIIIIIIIIIIIIIIIIIIIIIIIIIIIIIIIIIIIIIIIIIIIIIIIIIIIIIIIIIIIIIIIIIIIIIIIIIIIIIIIIIIIIIIIIIIIIIIIIIIIIIIIIIIIIIIIIIIIIIIIII 


\section{Introduction}

Corrosion of materials impacts the impedance coefficient $q$, which intervening in a Robin boundary condition, and identifying this coefficient turns out to be a way to locate the corroded part in a structure, and possibly evaluate the damage level, by an electric impedance tomography process. We are particularly interested in detecting corrosion on the internal wall of a tubular domain, standing for a pipeline for example.

Several models are presented in the literature. The corrosion effects may be seen as a loss of material modifying the geometry (see, for example, Kaup and Santosa [23], Inglese [20] etc). Focusing on the energy dispersion due to the local damage of the insulating film, Inglese and Fasino [19], give a simple model correlating the damage level to the transfert coefficient in a Robin boundary condition. Buttazo and Kohn [7] observe that the Robin boundary conditions can result from the introduction of a thin coating characterized by rapid oscillations. The potential model proposed by Vogelius and Xu [36] leads to a non linear relationship between the current flux and the potential, the simplest case of which brings us once again to the Robin boundary condition.

The mathematical setting of the problem is thus the following: Let $\Omega \subset \mathbb{R}^{2}$ be a connected or doubly-connected domain, the smooth boundary of which $\partial \Omega=\overline{\Gamma_{d}} \cup \overline{\Gamma_{u}}$ is made of two Jordan closed curves $\Gamma_{d}, \Gamma_{u}$ such that $\Gamma_{d} \cap \Gamma_{u}=\emptyset$. The electric potential $u$ solves the Laplace equation in $\Omega$, i.e.,

$$
\Delta u=0, \quad \text { in } \Omega .
$$

On $\Gamma_{d}$, both the Dirichlet and Neumann data of the electric potential $u$ are given, the first ones being provided by the measurements performed on this part of the boundary, and the second stand for the prescribed current flux.

$$
\begin{gathered}
u=u_{d}, \quad \text { on } \quad \Gamma_{d}, \\
\partial_{n} u=\phi, \quad \text { on } \quad \Gamma_{d}
\end{gathered}
$$

where $\partial_{n}$ stands for the partial derivative w.r.t. the outer normal unit vector to $\partial \Omega$.

As for the boundary condition on the unaccessible part of the boundary, we shall stick to the Robin model, as discussed by Santosa et al. [32]:

$$
\partial_{n} u+q u=0 \quad \text { on } \quad \Gamma_{u}
$$

where $q$ is the electrical impedance to be recovered.

The inverse problem we are interested in is to recover the unknown coefficient $q$ from the Cauchy data $u_{d}$ and $\phi$ on $\Gamma_{d}$. In the present paper, we shall focus on the design of robust identification algorithms. Robustness is understood as the sensibility with respect to noise in measured data. It is quite a different different issue from stability, though it is sometimes confused with it. Stability means continuity of the theoretical inverse problem 
solution with respect to the prescribed data, and it needs the data to remain in a space allowing the inverse problem to have a unique solution. Errors in data measurements are however not expected to behave this way. No matter how small the measurement errors are, there is usually no solution to the inverse problem related to the noisy data. One thus needs to solve a different problem, such as an optimization or a least squares one, which is equivalent to the original problem for exact data. The very issue to investigate is thus to figure out how close to the original inverse problem solution the one we compute that way is.

\section{An overview on uniqueness, stability and identification issues}

Up to our knowledge, the first identifiability result regarding the Robin inverse problem has been given by Inglese in [20], who proved in a 2D situation that a single measurement of $u$ on $\Gamma_{d}$ is enough to uniquely determine a positive coefficient $q$, provided $q \in \mathbb{C}_{0}^{3}$. This result has been improved in [8] in the $2 \mathrm{D}$ case, by extending the uniqueness result to continuous Robin coefficients $q$ with some appropriate negative lower bound. Some identifiability results for the non-linear heat equation can also be found in Choulli [14].

The stability issue deals with the continuous dependence of the unknown parameter on the measured data, which is a crucial issue for numerical applications, and has been the concern of many authors. Inglese [20] has proved the continuity, and the Gateaux differentiability of the direct map which associates to a given current flux $\phi$, the measured data on the boundary. Based on the use of differentiation with respect to the Robin parameter, a local and directional Lipschitz stability estimate is given in [8]. A Lispschitz monotone result is also given in the same paper. More recently, Sincich [33] has proved by a different way a global Lipschitz stability estimate in finite dimensional spaces of Robin coefficients, namely piecewise constant ones. As expected, because the global stability is well known to be no better than logarithmic, the Lipschitz constant in the above-mentioned estimate exponentially blow up as the Robin coefficients space dimension grows to infinity.

Actually, several authors have proved by various means the logarithmic global stability of the Robin inverse problem. Let us cite among them Fasino, Inglese [19], Alessandrini and Del Piero, Rondi [1], Choulli [14], Alessandrini and Sincich [2, 3], Chaabane, Fellah, Jaoua, Leblond [11], Leblond, Mahjoub, Partington [28]. In his already cited paper, Choulli [14] proves a local Lipschitz stability estimate for an arbitrary smooth domain, before establishing a $\log -\log$ stability estimate for rectangular domains.

As for the identification issue, most of the methods are use iterative algorithms. Kabanikhin and Karchevsky [22] solved this inverse problem by minimizing the trace error functional on $L^{2}\left(\Gamma_{d}\right)$ by a gradient iterative process. Klibanov and Santosa [24] used the quasi-reversibility method to solve the Cauchy problem for the Laplace's equation from which they derive an error estimate by using Carleman's inequalities. Slodi čka and Van Keer [34] proved an identification result based on the difference in the $L^{2}\left(\Gamma_{u}\right)$-sense between the outside and inside temperature on $\Gamma_{u}$. In [35], Slodička and Van Keer use 
the fact that the direct problem is linear wrt $u$ and apply the linear superposition principle in oredr to built the unknown trace of $u$ on the inaccessible boundary part $\Gamma_{u}$ using the overabundant information on the measurement part $\Gamma_{d}$. Inglese [20] has proposed an identification algorithm of the Robin coefficient based on an asymptotic developpement of the Robin function with respect to the width of the plate, leading to a thin plate approximation of the corrosion coefficient. Further, Fasino and Inglese [19] have proposed a Galerkin approximation for the Robin parameter by a trigonometric polynomial obtained by minimization procedure.

In a recent work, Louati [29] has proposed a direct (non-iterative) technique for detecting corrosion in pipelines from voltage-to-current observations. This algorithm is a MUSIC-type (multiple signal classification) one and it is based on an accurate asymptotic representation formula for the steady state current perturbations.

Any numerical identification method needs to overcome by some way or other the intrinsic instability of the Cauchy data completion problem, which is basically the one we explicitly or implicitly solve when aiming to recover the Robin coefficient, by some regularization technique. This consists in adding to the data misfit term some additional term whose role is to prevent instabilities from showing up. The main advantage of the fading regularization method proposed by Cimetière et al. [15], is that this additional term, which is the distance between two successive iterates on the completion part of the boundary, is swept away as convergence goes along. Strong convergence on the completed data however need to implement higher order versions of the algorithm, and though showing excellent numerical robustness, this features is not so far theoretically proved.

The numerical algorithm based on an energetic least-squares proposed in [8], and based on an idea by Kohn and Vogelius [25, 26], turns out to be quite general a method,and moreover self-regularizing. The method actually relies on the fact that the overabundance of boundary data on $\Gamma_{d}$ allows to solve two boundary value problems, a Neumann-Robin one and a Dirichlet-Robinn one, for any prescribed Robin coefficient. The solutions of the two of these problems coincide for the actual impedance. Choosing the energy gap between the two solutions as a misfit function to minimize is thus quite a natural idea. Beside offering a mathematically convenient function, that can be easily handled for example to differentiate, it also provides - because being distributed on the whole domain instead of the measurement boundary - with costless stabilization effects. The Kozlov alternated directions method [27], such as numerically exploited by A. Ben Abda et al. [4] to solve several inverse problems (recovery of a heat field on the internal wall of a pipe, identification of interface cracks, etc.) is based on the same idea.

An alternative approach has been successfully developed during the past decade. It consists to construct analytic approximations to solve the Cauchy problem, which is turned into a bounded extremal problem in a hardy space. Such a construction needs an explicit asymptotic expansion of the analytic approximant, and it needs to determine by some appropriate procedure the actual bound of that approximant on the unknown part of the boundary in order to stabilize the whole algorithm. These extended Cauchy data are then used in order to compute the Robin coefficient (see [12, 21]), and robustness of the whole process with respect to measurement errors is proved. 


\section{The Kohn and Vogelius algorithm}

Let $\Omega=\mathbb{D}$ such that: $\partial \Omega=\mathbb{T}=\overline{\Gamma_{u}} \cup \overline{\Gamma_{d}}$. The inverse problem $(\mathcal{I P})$ we are dealing with is the following:

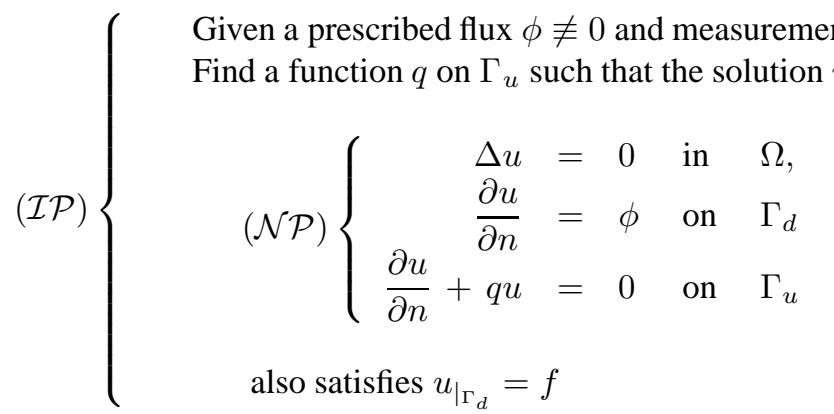

We shall assume that $\phi \in L^{2}\left(\Gamma_{d}\right)$ and $q$ belongs to the restricted set of admissible parameters $Q_{\text {ad }}$, defined by:

$$
Q_{\mathrm{ad}}=\left\{q \in W^{1,2}\left(\Gamma_{u}\right), \text { such that }\|q\|_{1, \gamma} \leq c \text { and } q \geq c^{\prime} \chi_{K}\right\}
$$

where $c$ and $c^{\prime}$ are two positive constants, and $K$ is a nonempty connected open subset of $\Gamma_{u}$ such that $\Gamma_{u} \cap K=\emptyset$. Though uniqueness is proved in the almost largest possible set of impedances, this restriction is necessary to prove stability and robustness.

For $q \in Q_{\text {ad }}$, we shall denote by $u^{D}(q, f)$ the solution of the following RobinDirichlet problem $(\mathcal{D P})$ using the measurements $f$ as a Dirichlet data:

$$
(\mathcal{D P})\left\{\begin{aligned}
\Delta u & =0 & & \text { in } \Omega \\
u & =f & & \text { on } \Gamma_{d} \\
\frac{\partial u}{\partial n}+q u & =0 & & \text { on } \Gamma_{u}
\end{aligned}\right.
$$

and by $u^{N}(q)$ the solution of the Neumann problem $(\mathcal{N} P)$ associated to $q$. As has been above, given any $q$, one can solve the two of these boundary value problems. These solutions coincide for the actual $q$, i.e. the one solving the inverse problem, that will be denoted $\bar{q}$. The cost function $J$ defined below is actually the energy gap between $u^{N}(q)$ and $u^{D}(q)$ :

$$
J(q)=\int_{\Omega}\left|\nabla u^{N}(q)-\nabla u^{D}(q, f)\right|^{2}+\int_{\Gamma_{u}} q\left|u^{N}(q)-u^{D}(q, f)\right|^{2}
$$

Minimizing it appears as a suitable way to capture the inverse problem solution. This leads to the following optimization problem:

$$
(\mathcal{O P})\left\{\begin{array}{l}
\text { Find } \bar{q} \in Q_{\mathrm{ad}} \text { such that } \\
J(\bar{q}) \leqslant J(q) \quad \forall q \in Q_{\mathrm{ad}} .
\end{array}\right.
$$


Referring to [8], the function $J$ has indeed a unique minimum, which is nothing but the solution $\bar{q}$ of the inverse problem $(\mathcal{I P})$.

\subsection{Some features of the optimization problem}

The first interesting feature of the optimization problem is that it still has a solution [9], which is not proved to be unique, even when the inverse problem fails to have one, for example in case the data lose compatible because of measurements errors.

However, a questioning raises about the sense of a solution to such a problem, which links to the sought solution of the inverse problem obviously sinks with this latter.

\subsubsection{Stability of the optimization problem}

The 'stability' result of the optimization problem we are dealing with in the present section actually extends this relationship to uncompatible prescribed data, and thus gives an answer: provided the 'measured data' are close to the actual ones, so will be any computed solution of the optimization problem to the inverse problem solution. Even though this continuity result still lacks a stability estimate, it somehow legitimates the algorithm process, which is to proceed looking at $(\mathcal{O P})$ instead of $(\mathcal{I P})$ even when this latter collapses because of erroneous data.

Theorem 3.1 (Stability) [9]

Let $\left(f_{n}\right)_{n \in \mathbb{N}}$ be a sequence of measurements in $W^{\frac{1}{2}, 2}\left(\Gamma_{d}\right)$, such that

$$
\lim _{n \rightarrow \infty}\left\|f_{n}-f\right\|_{\frac{1}{2}, 2, \Gamma_{d}}=0
$$

Then, $q_{n}$ being any solution to the perturbated optimization problem $\left(\mathcal{O} \mathcal{P}_{n}\right)$ with $f_{n}$ as input data, we have:

$$
\lim _{n \rightarrow \infty}\left\|q_{n}-\bar{q}\right\|_{L^{2}\left(\Gamma_{u}\right)}=0
$$

Proof (sketch): We first prove that the perturbated optimization problem $\left(\mathcal{O} P_{n}\right)$ has at least one solution $q_{n}$. Picking any of these, we then establish that the sequence $\left(q_{n}\right)_{n \in \mathbb{N}}$ is bounded in $H^{1}\left(\Gamma_{u}\right)$ and moreover satisfies:

$\lim _{n \rightarrow \infty} J\left(q_{n}\right):=\lim _{n \rightarrow \infty} \int_{\Omega}\left|\nabla u^{N}\left(q_{n}\right)-\nabla u^{D}\left(q_{n}, f_{n}\right)\right|^{2}+\int_{\Gamma_{u}} q_{n}\left|u^{N}\left(q_{n}\right)-u^{D}\left(q_{n}, f_{n}\right)\right|^{2}=0$.

Let then $\mu$ be an accumulation point of the real bounded sequence $\left\|q_{n}-\bar{q}\right\|_{L^{2}\left(\Gamma_{u}\right)}$. Then, there exists $\underline{q} \in \Phi_{a d}$ and a subsequence of $q_{n}$ still denoted by $q_{n}$ such that:

$$
\left\{\begin{array}{l}
\lim _{n \rightarrow \infty}\left\|q_{n}-\bar{q}\right\|_{L^{2}\left(\Gamma_{u}\right)}=\mu \\
q_{n} \rightarrow \underline{q} \text { weakly in } H^{1}\left(\Gamma_{u}\right) \\
q_{n} \rightarrow \underline{q} \text { strongly in } L^{2}\left(\Gamma_{u}\right)
\end{array}\right.
$$

According to (5) and the continuity of the mapping: 


$$
\begin{aligned}
\eta: L^{2}\left(\Gamma_{u}\right) & \longrightarrow H^{1}(\Omega) \\
q & \longmapsto u^{N}(q)
\end{aligned}
$$

we obtain:

$$
\lim _{n \rightarrow \infty} u^{N}\left(q_{n}\right)=\lim _{n \rightarrow \infty} u^{D}\left(q_{n}, f_{n}\right)=u^{N}(\bar{q})
$$

Moreover, $f_{n} \longrightarrow f$ in $H^{\frac{1}{2}}\left(\Gamma_{d}\right)$, and therefore:

$$
u^{N}(\underline{q})_{\mid \Gamma_{d}}=f .
$$

$\underline{q}$ then solves the inverse problem $(\mathcal{I P})$, which by uniqueness yields $\underline{q}=\bar{q}$. Consequently, $\bar{\mu}=0$ and

$$
q_{n} \longrightarrow \bar{q} \text { in } L^{2}\left(\Gamma_{u}\right)
$$

\subsubsection{Robustness}

The robustness of the process is a different story: erroneous data are not expected to lay in $W^{\frac{1}{2}, 2}\left(\Gamma_{d}\right)$, since noise is not smooth. Actually, noisy data can be written as follows:

$$
f^{\varepsilon}=f+\varepsilon
$$

where $f$ are the exact data and $\varepsilon \in L^{\infty}\left(\Gamma_{d}\right)$ such as:

$$
\|\varepsilon\|_{L^{\infty}\left(\Gamma_{d}\right)} \leq \epsilon
$$

where $\epsilon \in \mathbb{R}_{+}$is the noise level.

The point is that we are unable to define what a solution to the optimization problem with such unsmooth data might be. The data need to be smoothed before proceeding, which is actually the way most numerical algorithms deal with measurements. Using to that end the popular cubic B-splines, which are piecewise cubic functions that are twice continuously differentiable, one may smooth $f^{\varepsilon}$ and get $\tilde{f}^{\varepsilon} \in W^{\frac{1}{2}, 2}\left(\Gamma_{d}\right)$, thus making $(\mathcal{O P})$ have solutions among which we can pick $\tilde{q}^{\varepsilon}$.

Still, how close to the actual data are the smoothed ones ? Denoting by $h$ the splining path $h$ and assuming $f \in \mathcal{C}^{2}\left(\Gamma_{d}\right)$, we get the following estimates (see [10]):

$$
\begin{aligned}
& \left\|\tilde{f}^{\varepsilon}-f\right\|_{L^{\infty}\left(\Gamma_{d}\right)} \leq c_{1}\left(\epsilon+h^{2}\right) \\
& \left\|\left(\tilde{f}^{\varepsilon}-f\right)^{\prime}\right\|_{L^{\infty}\left(\Gamma_{d}\right)} \leq c_{2}\left(\frac{\epsilon}{h}+h\right)
\end{aligned}
$$

These error estimates inform us on the "'best smoothing path"' (with respect to the noise level $\epsilon$ ) to choose when using cubic B-splines:

$$
h \simeq \sqrt{\epsilon}
$$

Doing so, we obtain :

$$
\begin{array}{lll}
\|\tilde{f} \varepsilon-f\|_{L^{\infty}\left(\Gamma_{d}\right)} & =O(\epsilon) \\
\left\|(\tilde{f} \varepsilon-f)^{\prime}\right\|_{L^{\infty}\left(\Gamma_{d}\right)} & = & O(\sqrt{\epsilon})
\end{array}
$$


Theorem 3.2 (Robustness) [17]

Let $\left(f_{\varepsilon}\right)=f+\varepsilon$ stand for noisy measurements with $\varepsilon \in L^{\infty}\left(\Gamma_{d}\right)$ and $\|\varepsilon\|_{0, \infty, \Gamma_{d}} \leq \epsilon$. Let $\left(\tilde{f}_{\varepsilon}\right)$ be the data smoothed by using cubic B-splines with an appropriate path $(h \simeq \sqrt{\epsilon})$. Therefore, picking any solution $\left(\tilde{q}_{\varepsilon}\right)$ of the related optimization problem $(O P)$ problem with input data $\tilde{f}_{n}$, we have:

$$
\lim _{\epsilon \rightarrow 0}\left\|\tilde{q}_{n}-\bar{q}\right\|_{L^{2}\left(\Gamma_{u}\right)}=0
$$

Proof: This is actually a straightforward consequence of the above stability theorem, and to the error estimates (7). Indeed, by interpolation, we get from these estimates:

$$
\left\|\tilde{f}^{\varepsilon}-f\right\|_{\frac{1}{2}, \Gamma_{d}}=O\left(\epsilon^{\frac{3}{4}}\right)
$$

which means that $\lim _{\epsilon \rightarrow 0}\left\|\tilde{f}^{\varepsilon}-f\right\|_{\frac{1}{2}, 2, \Gamma_{d}}=0$. The splined noisy data $\tilde{f}^{\varepsilon}$ may thus be seen as an approximation of the exact data $f$. Applying the above stability Theorem 3.1 to these input data immediately yield the announced robustness result.

\subsection{Numerical results}

Aiming to solve the optimization problem using a gradient method, we are interested in computing the Gâteaux-derivative of the cost function $J$ with respect to the unknown Robin coefficient $q$. Thanks to [8], we are able to compute the gradient of the cost function without needing to solve an adjoint problem solution, the result being the following: (see [8]):

$$
J^{\prime}(q) \cdot q^{\prime}:=\lim _{h \rightarrow 0^{+}} \frac{J\left(q+h q^{\prime}\right)-J(q)}{h} \int_{\Gamma_{u}} q^{\prime}\left[\left(u^{D}(q, f)\right)^{2}-\left(u^{N}(q)\right)^{2}\right]
$$

The gradient algorithm applied to the Kohn and Vogelius cost function thus writes down as follows: 


\section{The Kohn \& Vogelius algorithm:}

1) Initialization : Choose an initial guess $q_{0}$ in the approximation space, and a step size $\rho>0$;

2) Iteration : $q_{k}$ being computed,

a) Compute the solutions of $u_{k}^{N}$ and $u_{k}^{D}$ of the Neumann and Dirichlet problems $\left(P_{k}^{N}\right)$ and $\left(P_{k}^{D}\right)$ related to $q_{k}$

b) Compute the gradient of $\mathcal{J}$ at $q_{k}$, using the following formula giving the derivative of $\mathcal{J}$ in any direction $\psi$ :

$$
\nabla \mathcal{J}\left(q_{k}\right) \cdot \psi=\int_{\Gamma_{u}} \psi\left[\left(u_{k}^{D}\right)^{2}-\left(u_{k}^{N}\right)^{2}\right]
$$

c) Update the Robin coefficient by:

$$
q_{k+1}=q_{k}-\rho \nabla \mathcal{J}\left(q_{k}\right)
$$

3) Stop test: If $\frac{\left|q_{k+1}-q_{k}\right|}{\left|q_{k}\right|}$ is smaller than a prescribed threshold, then stop, else return to step (2) with $k=k+1$

Experimental measurements have been simulated by synthetic data obtained by means of numerical computations, solving the forward problem $(\mathcal{N} \mathcal{P})$. All the numerical experiments have been carried out on the unit disc, with $\Gamma_{u}=\left\{e^{i \theta} ; \theta \in\right] 0, \frac{\pi}{2}[\}$. The forward problems have been solved by quadratic finite elements.

Several options are available to represent the approximated impedance (Robin coefficient). One of them is to use the finite element shape functions, not necessarily those used to computate the states $u^{D}$ and $u^{N}$. It is well known however that, aiming to solve inverse problems, the unknowns to recover should preferably not be too numerous. A hierarchic approach, gradually enriching the representation together with smoothing, can make up for this limit. Though successful for smooth impedances recovery, this method however fails when it comes to capture oscillating ones. The reason is that the gradient components of the cost function with respect to the higher frequencies basis functions (for instance cosines) are:

$$
\frac{\partial J}{\partial \varphi}(\cos (2 j \theta))=\nabla J(\varphi) \cos (2 j \theta)=\int_{0}^{\frac{\pi}{2}} \cos (2 j \theta)\left[\left(u^{D}\right)^{2}-\left(u^{N}\right)^{2}\right] d \theta
$$

This is nothing but the $j-t h$ Fourier coefficient of $\left[\left(u_{D}\right)^{2}-\left(u_{N}\right)^{2}\right]$, which all the more fastly decays to zero that this function is smooth. The gradient algorithm applied to the Kohn \& Vogelius cost function turns out to only seek descent along the lower frequencies, damping down the higher order components. If aiming to capture these, one should thus better prefer a relaxation method, which successively minimizes along each of the frequencies without making them compete, rather than the gradient one. This needs to preferably represent the impedance in a Fourier basis brings an additional improvement (see Figure 1), which indeed has been noticed to provide a significant improvement with 
respect to the finite element representation.

Still, this is not enough to capture the higher order oscillations. The method actually shows its amazing regularization power, by its controlling the instabilities that show up in the higher order components. Slight improvements can be brought by enhancing - however heuristically - the so dampened frequencies, or by giving to the Kohn and Vogelius function the role of a regularizer instead of that of the cost function itself. An accurate capture of the oscillations however definitely needs a specifically designed method to that end (see Figure 1), such as those used in image processing [5] in order to recover the textures.

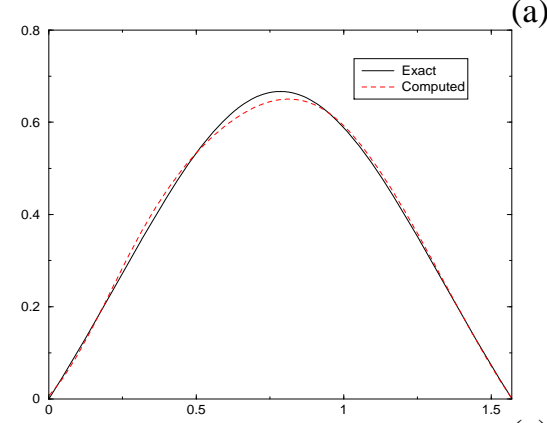

(a)

(c)

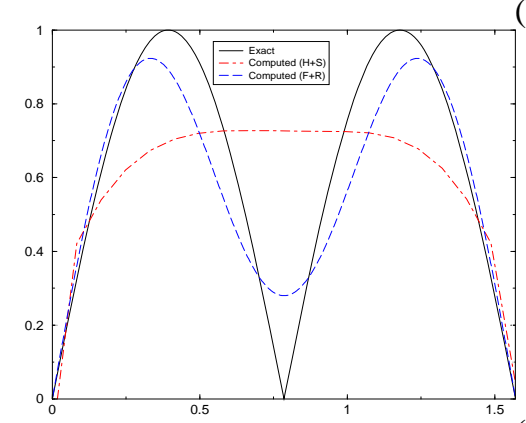

(b)
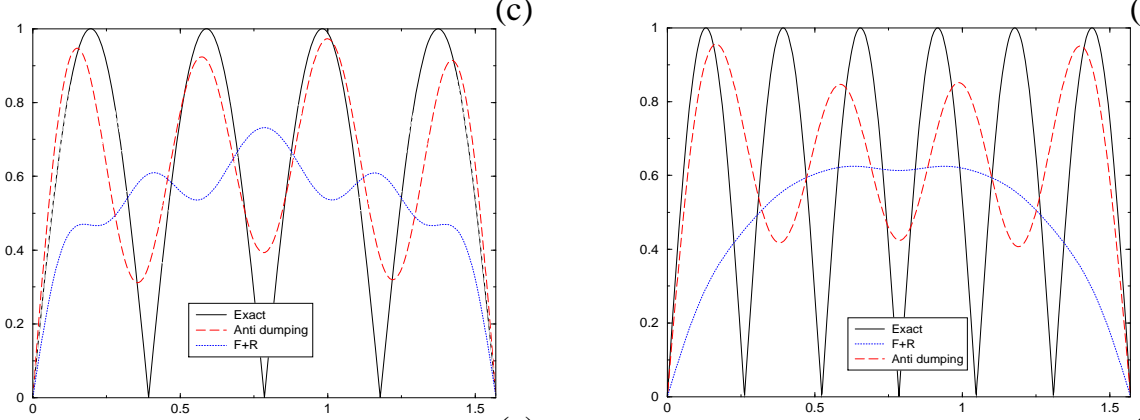

(d)

(e)
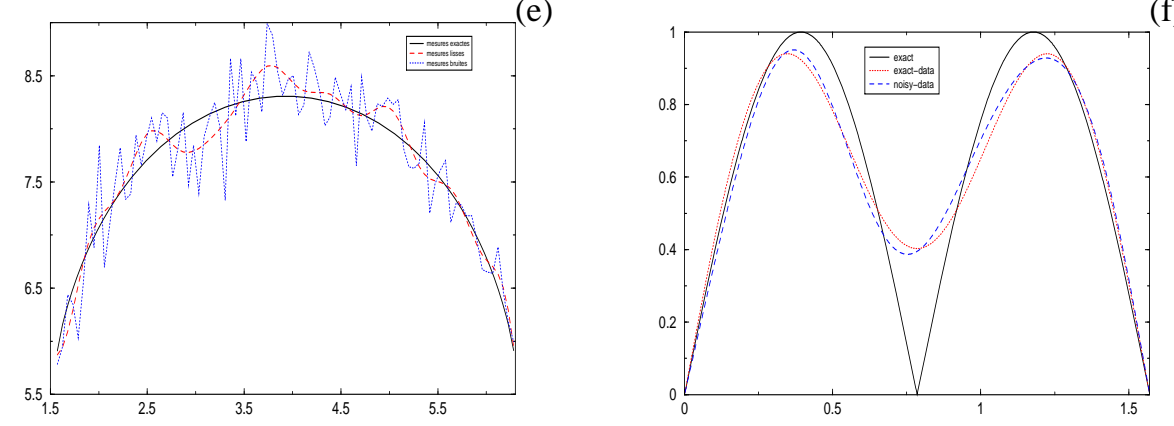

(f)

Figure 1. (a) Reconstruction by piecewise linear representation: hierarchic with smoothing, (b) reconstruction on a Fourier basis: relaxation method, (c, d) Capturing oscillations by anti-damping (Fourier + relaxation), (e, f) Reconstruction using $10 \%$ noisy data. 


\section{Algorithms based on analytic extensions}

In this section, $\Omega$ is the annulus $\Omega=\mathbb{D} \backslash s \mathbb{D}$ for some fixed $s$ with $0<s<1$, where $\mathbb{D}$ is the unit disc and $\mathbb{T}$ the unit circle. Omega stands here for the cross section of a pipe. Up to a conformal mapping, all the results here presented can be extended to doubly connected domain in $\mathbb{R}^{2}$. Consequently, $\partial \Omega=\mathbb{T} \cup s \mathbb{T}$.

Let $\Gamma_{d}$ be a non-neglectible measurable subset of $\mathbb{T}$, and $\Gamma_{u}=\partial \Omega \backslash \Gamma_{d}$. In order to recover both Dirichlet and Neumann boundary data on the internal boundary of an annulus, and then compute the Robin coefficient which is actually the quotient of these data described here, we shall use the theory of analytic approximations by solving "bounded extremal problems" in Hardy spaces [6, 13].

Indeed, let $\phi \in L^{2}\left(\Gamma_{d}\right)$ and assume that $q \in Q_{\text {ad }}$, then $u_{\left.\right|_{\partial \Omega}} \in W^{1,2}(\partial \Omega)$. Then, from the Cauchy-Riemann equations, there exists a function $v$ harmonic in $\Omega$ such that $\partial_{\theta} v=\partial_{n} u$ on $\partial \Omega$, where $\partial_{\theta}$ stands for the tangential partial derivative on $\partial \Omega$. Hence, $v$ is given on $\Gamma_{d}$ up to a constant by

$$
v_{\left.\right|_{\Gamma_{d}}}\left(e^{i \theta}\right)=\int_{\theta_{0}}^{\theta} \phi\left(e^{i \tau}\right) d \tau
$$

The harmonic conjugate operator is bounded in $L^{2}(\partial \Omega)$, whence $v_{\mid \partial \Omega} \in W^{1,2}(\partial \Omega)$. Thus, $f=u+i v$ is analytic in $\Omega$ and $f_{\left.\right|_{\partial \Omega}} \in W^{1,2}(\partial \Omega)$ is given on $\Gamma_{d}$ by:

$$
f\left(e^{i \theta}\right)=u_{d}\left(e^{i \theta}\right)+i \int_{\theta_{0}}^{\theta} \phi\left(e^{i \tau}\right) d \tau
$$

Then on $\Gamma_{u}$, we have

$$
q=-\frac{\partial_{\theta} v}{u}-\frac{\partial_{\theta} \operatorname{Im} f}{\operatorname{Ref}}
$$

which gives the link to be used between $q$ and $f$, in order to recover $q$ from approximations to $f$ on $\Gamma_{d}$ of the boundary $\partial \Omega$.

Let us introduce here the Hardy space $H^{2}(\mathbb{D})$ of analytic functions in the unit disk $\mathbb{D}$ whose $L^{2}$ norms on the unit circle $\mathbb{T}$ are bounded [16]. Let $\bar{H}_{0}^{2}(s \mathbb{D})$ be the Hardy space consisting of the analytic functions on the complement of $s \bar{D}$ that have boundary values in $L^{2}(\partial \Omega)$ and vanish at infinity.

From the above-mentioned regularity properties, the function $f$ is bounded in $L^{2}\left(\Gamma_{d}\right)$, and we then seek an (approximate) extension of $f$ in the so-called Hardy space denoted by $H^{2}(\Omega)=H^{2}(\mathbb{D}) \oplus \bar{H}_{0}^{2}(s \mathbb{D})$ defined in [31]. It is also possible to define the Hardy spaces $H^{2}(\partial \Omega)$, as the closure in $L^{2}(\partial \Omega)$ of the set $R_{\Omega}$ of rational functions whose poles lie in $\mathbb{C} \backslash \bar{\Omega}$. The spaces $H^{2}(\Omega)$ and $H^{2}(\partial \Omega)$ are then isomorphic in a natural way, and so we identify the two spaces, see [13].

For $m \geq 1$, introduce $H^{m, 2}=H^{2}(\partial \Omega) \cap W^{m, 2}(\partial \Omega)$, the Hardy-Sobolev space of the annulus $\Omega$. So, a function $f \in H^{m, 2}$ has the following expansion: 


$$
f(z)=\sum_{n \in \mathbb{Z}} f_{n} z^{n} \quad \text { for } \quad z \in \Omega, \quad \text { where }\|f\|_{W^{m, 2}(\partial \Omega)}^{2} \sum_{n \in \mathbb{Z}} l_{m, n}\left|f_{n}\right|^{2}
$$

where

$$
\left\{\begin{array}{l}
l_{m, n}=w_{m, n}+\mu_{m, n} s^{2 n}, \quad \text { with } \\
w_{m, n}=1+\sum_{k=1}^{m} \prod_{q=0}^{k-1}(n-q)^{2} \quad \text { and } \quad \mu_{m, n}=1+\sum_{k=1}^{m} s^{-2 k} \prod_{q=0}^{k-1}(n-q)^{2} .
\end{array}\right.
$$

\subsection{Approximation in Hardy-Sobolev classes}

Suppose now we are given $f \in L^{2}\left(\Gamma_{d}\right)$ and we wish to approximate $f$ as well as possible by the restriction to $\Gamma_{d}$ of an $H^{2}(\partial \Omega)$ function, i.e., $g_{\left.\right|_{\Gamma_{d}}}$ for $g \in H^{2}(\partial \Omega)$. In view of the results established in $[6,13]$, the space $H^{2}(\partial \Omega)_{\left.\right|_{\Gamma_{d}}}$ is dense in $L^{2}\left(\Gamma_{d}\right)$. Then, there exists a sequence $\left(g_{n}\right)$ of $H^{2}(\partial \Omega)$ functions such that $\left\|g_{\left.n\right|_{\Gamma_{d}}}-f\right\|_{L^{2}\left(\Gamma_{d}\right)} \rightarrow 0$. However, if $f \neq g_{\left.\right|_{\Gamma_{d}}}$ for any $g \in H^{2}(\partial \Omega)$ then it follows that $\left\|g_{\left.n\right|_{\Gamma_{u}}}\right\|_{L^{2}\left(\Gamma_{u}\right)} \rightarrow \infty$, which means that the approximation problem is ill-posed without further constraint.

In order to determine an extension on $\Gamma_{u}$, and to prevent instability to show up, we thus impose a norm constraint, an upper-bound, to the approximation of $f$ on $\Gamma_{d}$. This motivates the following bounded extremal problem (BEP), which is a problem of analytic approximation of incomplete data in Hardy classes. To fix ideas, we consider the following (BEP) problem:

$$
\left\{\begin{array}{l}
\text { Given } f \in W^{m, 2}\left(\Gamma_{d}\right) \backslash H_{\mid \Gamma_{d}}^{m, 2}, \quad \text { and } \quad M>0 \\
\text { find a function } g \in H^{m, 2} \quad \text { such that }\|g\|_{W^{m, 2}\left(\Gamma_{u}\right)} \leq M \quad \text { and } \\
\|f-g\|_{W^{m, 2}\left(\Gamma_{d}\right)}=\inf \left\{\|f-\psi\|_{W^{m, 2}\left(\Gamma_{d}\right)}: \psi \in H^{m, 2},\|\psi\|_{W^{m, 2}\left(\Gamma_{u}\right)} \leq M\right\}
\end{array}\right.
$$

Recall that, in practice, $f$ is given by the available data from (9), whereas $\Gamma_{d}$ is the part where these data can be measured. The (BEP) problem has a unique solution $g_{m} \in H^{m, 2}$, which can expressed as follows [21, 30]:

$$
\begin{gathered}
g_{m}=\left(I+\lambda T_{m}\right)^{-1} P_{H^{m, 2}} \tilde{f}, \text { where } \\
\tilde{f}=\chi_{\Gamma_{d}}\left(f, \cdots, f^{(m)}\right) \text { and } T_{m} g=P_{H^{m, 2}} \chi_{\Gamma_{u}}\left(g, \cdots, g^{(m)}\right),
\end{gathered}
$$

for the unique $\lambda>-1$ such that $\left\|g_{m}\right\|_{W^{m, 2}\left(\Gamma_{u}\right)}=M$ if $f \notin H_{\Gamma_{d}}^{m, 2}$.

REMARK. - T

he Bounded Extremal Problem has also been studied for the unit disc $\mathbb{D}$ by Chaabane et al. [12] (for $m=0$ ). On the Fourier basis, the operator $T:=T_{0}$ is in that case a semi-infinite Toeplitz matrix $T_{k, l}=T_{k-l}, k, l \geq 0$. Whenever $\Gamma_{d}$ coincides with the arc $\left(e^{-i \theta_{0}}, e^{i \theta_{0}}\right), T$ can be expressed as:

$$
T_{k, l}=\frac{\sin (k-l) \theta_{0}}{\pi(k-l)}, \text { for } k \neq l, \quad T_{l, l} \frac{\theta_{0}}{\pi} .
$$


Now, we shall be concerned with continuity properties of the solutions $g_{m}$ with respect to the data $f$ and $M$, see $[21,28,30]$. Let $\Psi_{m}$ be the mapping defined by:

$$
\begin{aligned}
\Psi_{m}: W^{m, 2}\left(\Gamma_{d}\right) \times \mathbb{R}_{+}^{*} & \longmapsto H^{m, 2} \\
(f, M) & \longmapsto g_{m}(f, M),
\end{aligned}
$$

where $g_{m}(f, M)$ the solution for $H^{m, 2}$ associated to the data $f$ and $M$.

Let $\mathcal{D}_{m}=\left\{(h, M) \in H_{\mid \Gamma_{d}}^{m, 2} \times \mathbb{R}_{+}^{*} \quad\right.$ tq $\left.\|h\|_{W^{m, 2}\left(\Gamma_{u}\right)}<M\right\}$.

Theorem 4.1 (Continuity with respect to the prescribed data and the bound) The mapping $\Psi_{m}$ is continuous on $\left(W^{m, 2}\left(\Gamma_{d}\right) \times \mathbb{R}_{+}^{*}\right) \backslash \mathcal{D}_{m}$, with respect to the strong topology. Moreover, on $W^{m, 2}\left(\Gamma_{d}\right) \times \mathbb{R}_{+}^{*}$, the mapping $\Psi_{m}$ is continuous with respect to the weak topology of $H^{m, 2}$ while $P_{W^{m, 2}\left(\Gamma_{d}\right)} \Psi$ is a strongly continuous mapping into $W^{m, 2}\left(\Gamma_{d}\right)$. Thus, $\Psi_{m}$ is continuous with respect to the strong topology of $H^{m-1,2}$.

Proof (Sketch): Let us start with $m=0$. The first step in the proof consists to establish, for a given $f \in L^{2}\left(\Gamma_{d}\right), f \notin H^{2}\left(\Gamma_{d}\right)$ the continuity of the error mapping $e_{f}$ defined by:

$$
\begin{aligned}
e_{f}: & \mathbb{R}_{+}^{*} \rightarrow \mathbb{R}_{+} \\
& M \mapsto\|g(f, M)-f\|_{L^{2}\left(\Gamma_{d}\right)},
\end{aligned}
$$

after having proved that its convexity and its decay to zero when $M \rightarrow \infty$.

Next, $\left(f_{n}\right)$ being a sequence in $L^{2}\left(\Gamma_{d}\right)$ such that $\left\|f_{n}-f\right\|_{L^{2}\left(\Gamma_{d}\right)} \rightarrow 0$ and choosing a sequence $\left(M_{n}\right)$ in $\mathbb{R}_{+}^{*}$ such that $M_{n} \rightarrow M$, we prove that:

$$
\lim _{n \rightarrow \infty} e_{f_{n}}\left(M_{n}\right)=e_{f}(M) .
$$

On the other hand, the sequence $\left(g\left(f_{n}, M_{n}\right)\right)$ being bounded in $H^{2}$, there exists a subsequence that weakly converges there to some $\tilde{g} \in H^{2}(\Omega)$. Since the solution to (BEP) is unique (thanks to the strict convexity of the norm), we necessarily have that $\tilde{g}=g(f, M)$. This proves the weak convergence of $\left(g\left(f_{n}, M_{n}\right)\right)$ to $(g(f, M))$ in $H^{2}(\Omega)$. Moreover, we deduce from (12) and $\left\|g\left(f_{n}, M_{n}\right)-f_{n}\right\|_{L^{2}\left(\Gamma_{d}\right)} \rightarrow\|g(f, M)-f\|_{L^{2}\left(\Gamma_{d}\right)}$ that strong convergence actually holds in $L^{2}\left(\Gamma_{d}\right)$.

Now, whenever $(f, M) \notin \mathcal{D}_{0}$, we get :

$$
\limsup _{n \rightarrow \infty}\left\|g\left(f_{n}, M_{n}\right)\right\|_{L^{2}\left(\Gamma_{u}\right)} \leq \limsup _{n \rightarrow \infty} M_{n}=M=\|g(f, M)\|_{L^{2}\left(\Gamma_{u}\right)},
$$

which yields, since we already have the weak convergence of $g\left(f_{n}, M_{n}\right)$ to $g(f, M)$ in $H^{2}(\Omega)$, its strong convergence on $\Gamma_{u}$.

Applying these results to the $m$ first derivatives of the function $g_{m}$ finally ends the proof. 
REMARK. - :

It might sound surprising that the strong convergence $\Gamma_{u}$ is only obtained for non analytic data. As a matter of fact, this only highlights the ill posedness of the analytic extension problem. Besides, there is a fundamental discontinuity in the (BEP) problem for analytic data : it has a unique solution provided the prescribed bound is large enough (actually larger than the actual one), otherwise it has none. Whereas for non analytic data, the (BEP) always has a unique solution, whatever the value of the bound is. Hopefully, real data (i.e. noisy ones, even smoothed) are not analytic, which is a chance for the forthcoming algorithmic construction.

\subsection{Robust algorithms based on (BEP) approximants}

Because experimental measurements or numerical data are attached to errors, the choice of the constraint $M$ on $\Gamma_{u}$ is crucial for the problem. In the case where $f_{\varepsilon}=f+\varepsilon$, with a non analytic perturbation $\varepsilon\left(\varepsilon \in W^{m, 2}\left(\Gamma_{d}\right)\right.$ and $\left.\varepsilon \notin H_{\left.\right|_{\Gamma_{d}}}^{m, 2}\right)$, and $f \in H^{m, 2}$, then the bound allowed to the data on $\Gamma_{u}$ is always saturated, which means that the extension process makes totally use of the the granted freedom on $\Gamma_{u}$ in order to better fit the prescribed data on $\Gamma_{d}$.

Let us denote by $M_{0}:=\|f\|_{W^{m, 2}\left(\Gamma_{u}\right)}$.

The continuity results of Theorem 4.1 indicate that if one wants to find a best approximant $g_{m}$, it is necessary to choose a constraint sufficiently close to $M_{0}\|f\|_{W^{m, 2}\left(\Gamma_{u}\right)}$. However, this constraint $M_{0}$ is an unknown of the problem, since it depends on the behaviour of $f$ on $\Gamma_{u}$, whereas measurements are made only on $\Gamma_{d}$.

There are several possible methods for determining a suitable value. One of them is the so-called "cross-validation" method, consisting to devote some part of the available data on $\Gamma_{d}$ in order to obtain an estimate for $M$ as in [12].

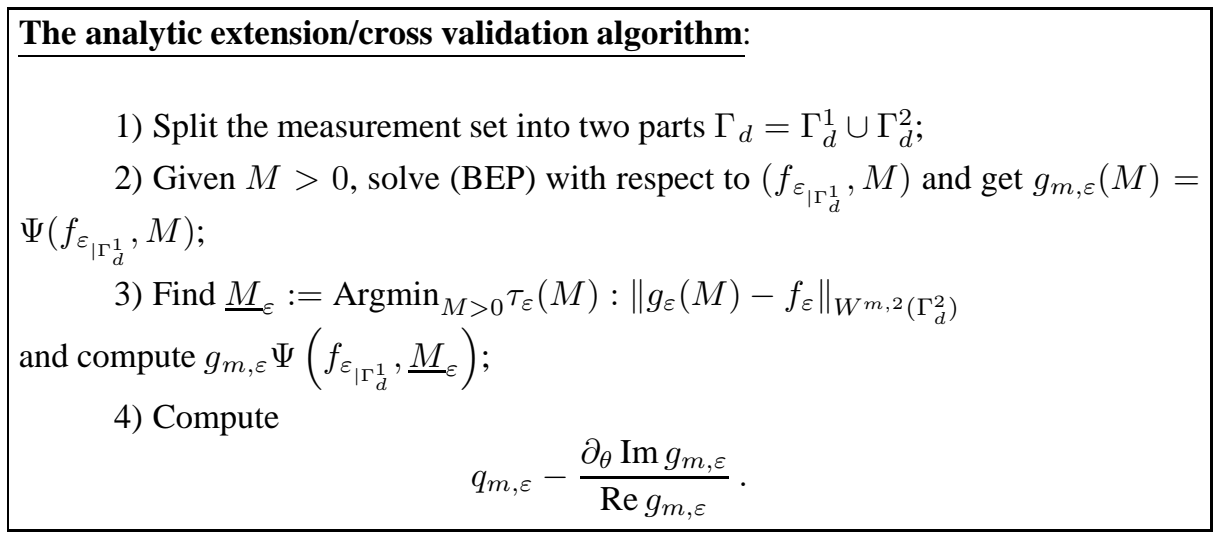

A less data consuming approach, not requiring to devote a part of them to the bound determination task, has been proposed and successively implemented in [21]. Whichever method is used to determine the bound, it should be stressed however that both tasks of 
recovering the bound and extending the data by solving a (BEP) need to be tackled in the same time. They are actually two inseparable parts of a single algorithm: an accurate estimate of the bound is needed to construct a proper approximation of the extended data, which in turn are the material the bound is computed from.

Let $\underline{c}, \bar{c}>0$ and define the following classes of "admissible" electrical impedances:

$$
\mathcal{Q}_{a d}^{m}=\left\{q \in \mathcal{C}^{m}\left(\Gamma_{u}\right) ;\left|q^{(k)}(x)\right| \leq \bar{c}, \quad 0 \leq k \leq m, \text { and } q(x) \geq \underline{c} \forall x \in \Gamma_{u}\right\}
$$

Therefore, we have the following continuity results:

Theorem 4.2 (Robustness) Suppose $\Phi \in W^{m-1,2}\left(\Gamma_{d}\right), q \in Q_{a d}{ }^{m-1}, m \geq 2$. Let then $f_{\varepsilon}=u_{d}+i \int \Phi d \theta+\varepsilon \in W^{m-1,2}\left(\Gamma_{d}\right)$ and $g_{m, \varepsilon}$ as above. As $\|\varepsilon\|_{W^{m-1,2}\left(\Gamma_{d}\right)} \rightarrow 0$ it holds that:

$$
\operatorname{Re} g_{m, \varepsilon} \rightarrow u \text { in } W^{m-1,2}(\partial \Omega), \partial_{\theta} \operatorname{Im} g_{m, \varepsilon} \rightarrow \partial_{n} u \text { in } W^{m-2,2}(\partial \Omega)
$$

Also

$$
q_{m, \varepsilon} \rightarrow q\left(\text { strongly) in } W^{m-2,2}\left(\Gamma_{u}\right)\right.
$$

Proof: This is an immediate consequence of Theorem 4.1 and if $g_{m}$ solve the $(\mathrm{BEP})_{m}$ problem for the data $f$ and the constraint $M$, then for all $k=0, \cdots, m$ the $k$-th derivative $g_{m}^{(k)}$ is the solution of (BEP) problem associate to the data $f^{(k)}$ and $M_{k}:=\left\|g_{m}^{(k)}\right\|_{L^{2}\left(\Gamma_{u}\right)}$.

The algorithm that has been described above in the present section is the $m$-th order one. From Theorems 4.1 and 4.2, we can notice that an additional regularity on the data, and a higher order method too, are required to get acceptable continuity results, i.e. strong convergence of $q_{m, \varepsilon}$ to $q$ in $W^{m-2,2}\left(\Gamma_{u}\right)$. The 1-st order method is the minimal one needed to get strong convergence of the extended data, whereas it requires up to the second order one to obtain strong convergence on the Robin coefficients in $L^{2}\left(\Gamma_{u}\right)$. The numerical results shown in the next section confirm this analysis.

REMARK. - A

s for the above Kohn and Vogelius algorithm of Section 3, the Theorem 4.1 result is not exactly a robustness one, since the noisy data $f_{\varepsilon}$ cannot be expected to hold the required regularity $W^{m-1,2}\left(\Gamma_{d}\right)$. Smoothing the noisy data prior to solving the analytic extension problem is therefore required. The estimates (7) actually show that the differentiated smoothed noisy data may be seen as approximations of the derivatives of the actual ones, with a noise level $\sqrt{\epsilon}$. This allows to reiterate the splining procedure with an appropriate path $h_{k}$ as many times as desired, thus obtaining smooth approximants of the $k-t h$ derivatives with an $O\left(\epsilon^{\frac{1}{2 k}}\right)$ error [10]. The so-splined noisy data, which satisfy the above theorem assumptions, are actually the ones that have been used as inputs in the analytic extension algorithm. 


\subsection{Numerical results}

In the numerical experiments we are presenting in this section, a numerical validation of the (BEP) reconstruction of the impedance $q$. Let us consider explicit data with a polar singularity $a$, resulting from:

$$
f(z)=12+\frac{2(z-1)}{z-a}, a \in s \mathbb{D} .
$$

We have considered both cases of full prescribed data (i.e., $\Gamma_{d}=\mathbb{T}$ ), and of incomplete data (i.e., data prescribed on a part of the outer boundary). The latter case is actually the most realistic one in the framework of non-destructive testing applications.

For $\Gamma_{d} \subsetneq \mathbb{T}, a=0.1$ and $s=0.6$, Figure 2 illustrates the accuracy of the method at order $m=1,2$ and for $M \simeq M_{0}$, as regards the analytic extension as well as the associated electrical impedance for noisy data. Noise has been generated by a random variable whose uniform norm ranges from $1 \%$ to $10 \%$ of $\|f\|_{\infty}$. As expected from the robustness results of the above subsection 4.2, the data extension process resists to noise better than the Robin coefficient recovery one does, although this latter is pretty robust. Also, we observe that the zero-order method $(a)$ is uneffective for the Robin coefficient recovery and for order 1 and 2, the reconstruction of $q$ shows ill behaviour on the extremes of the inner circle $s \mathbb{T}$. Indeed, the effect of noise is more important on the derivatives of the smoothed function $\tilde{f}^{\varepsilon}$ obtained by using cubic B-splines (see (6)-(7)).

Let us now study the behaviour of the (BEP) method for the reconstruction of oscillating impedances. First we are considering the case of full data $\left(\Gamma_{d}=\mathbb{T}\right)$. The data used are generated by the finite element computation of the forward problem with Neumann data $\phi=1$ on $\mathbb{T}$ and the exact Robin coefficient $q=\sin (k \theta)$ on $s \mathbb{T}$ for $k \geq 3$.

Figure 3 shows the sensitivity of the reconstruction method with respect to $k$, which parameterizes the number of oscillations. For full data, the reconstruction remains acceptable up to $k=8$, whereas it goes harsher beyond.

In Figure 4, two plots of the error are given, the first one with respect to the number of oscillations $k$ (full data on the outer boundary), and the second with respect to the amount of available data whenever these data are incomplete.

In case of incomplete data $\left(\Gamma_{d} \subsetneq \mathbb{T}\right)$, the second plot Figure 4 shows that the error on the Robin coefficient remains acceptable for a rather small quantity of available data - actually half of the outer boundary is enough - and the error decreases quite fast with respect to the increase of prescribed data.

\section{Comparison}

In this section we are briefly comparing the numeric behaviour of the two of the algorithms here presented and studied. The cross section $\Omega$ is an annular thick domain with radii $r_{1}=1$ and $r_{2}=0.6$. The internal boundary is denoted $s \mathbb{T}$ on which the data are lacking and the external one $\mathbb{T}$ on which the data are overspecified. For the example the data used are generated by the finite element computation of the forward problem with Neumann data $\phi=1$ on $\mathbb{T}$ or $\Gamma_{d} \subsetneq \mathbb{T}$ and the exact Robin coefficient $\varphi=|\sin (k \theta)|$ 

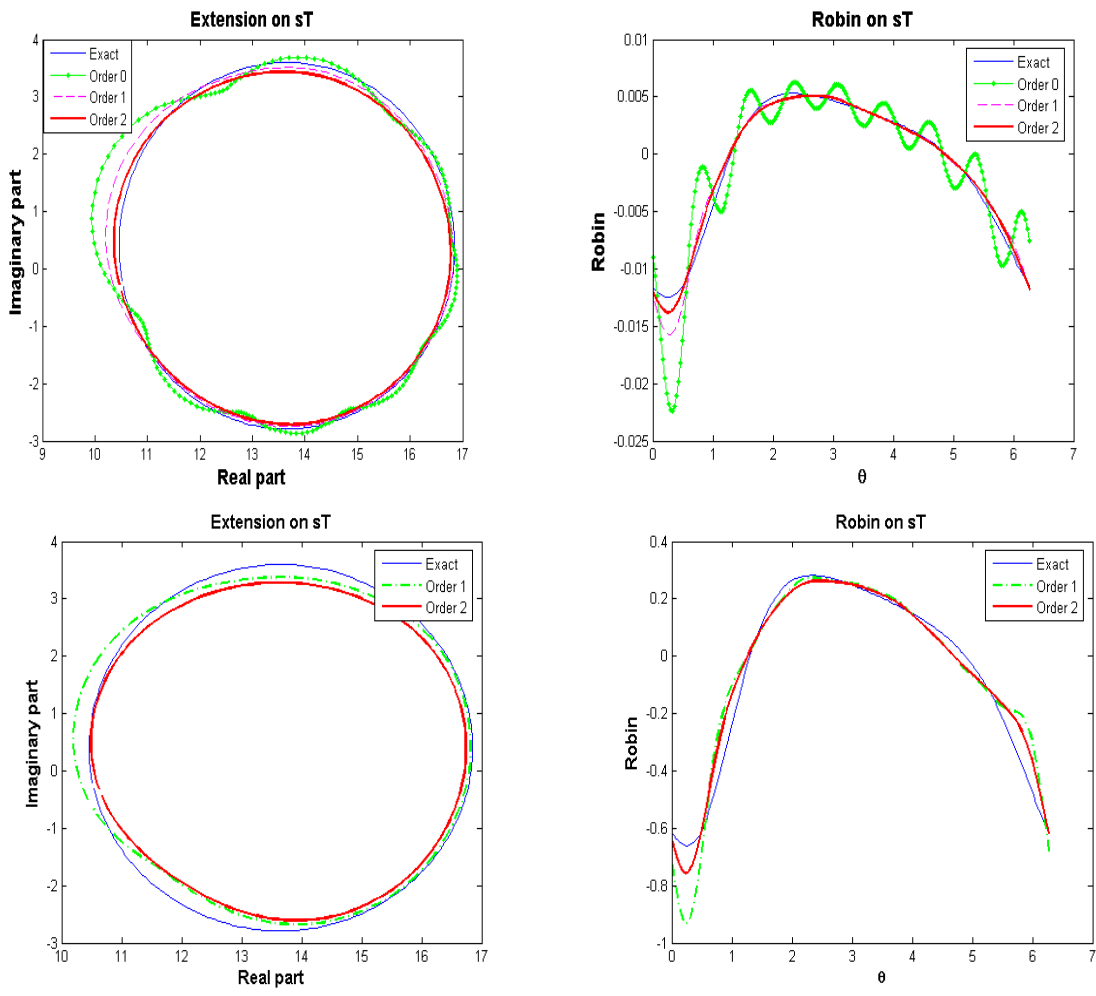

(a)
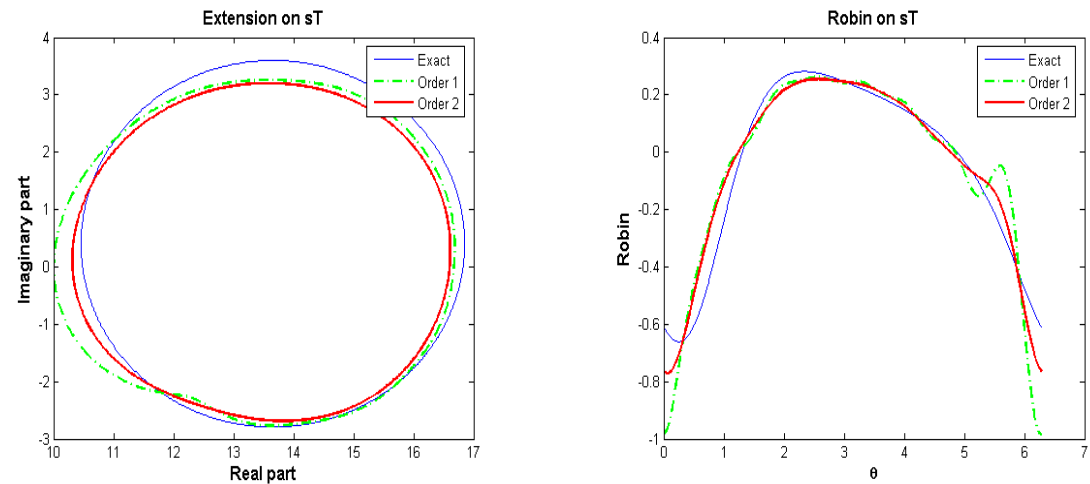

Figure 2. Recovery from noisy data (1\%, $5 \%, 10 \%$ noise, methods of order zero $(a)$ and of order 1, 2)-bounded extensions (left) and electrical impedances $q$ (right) on $s \mathbb{T}$. 

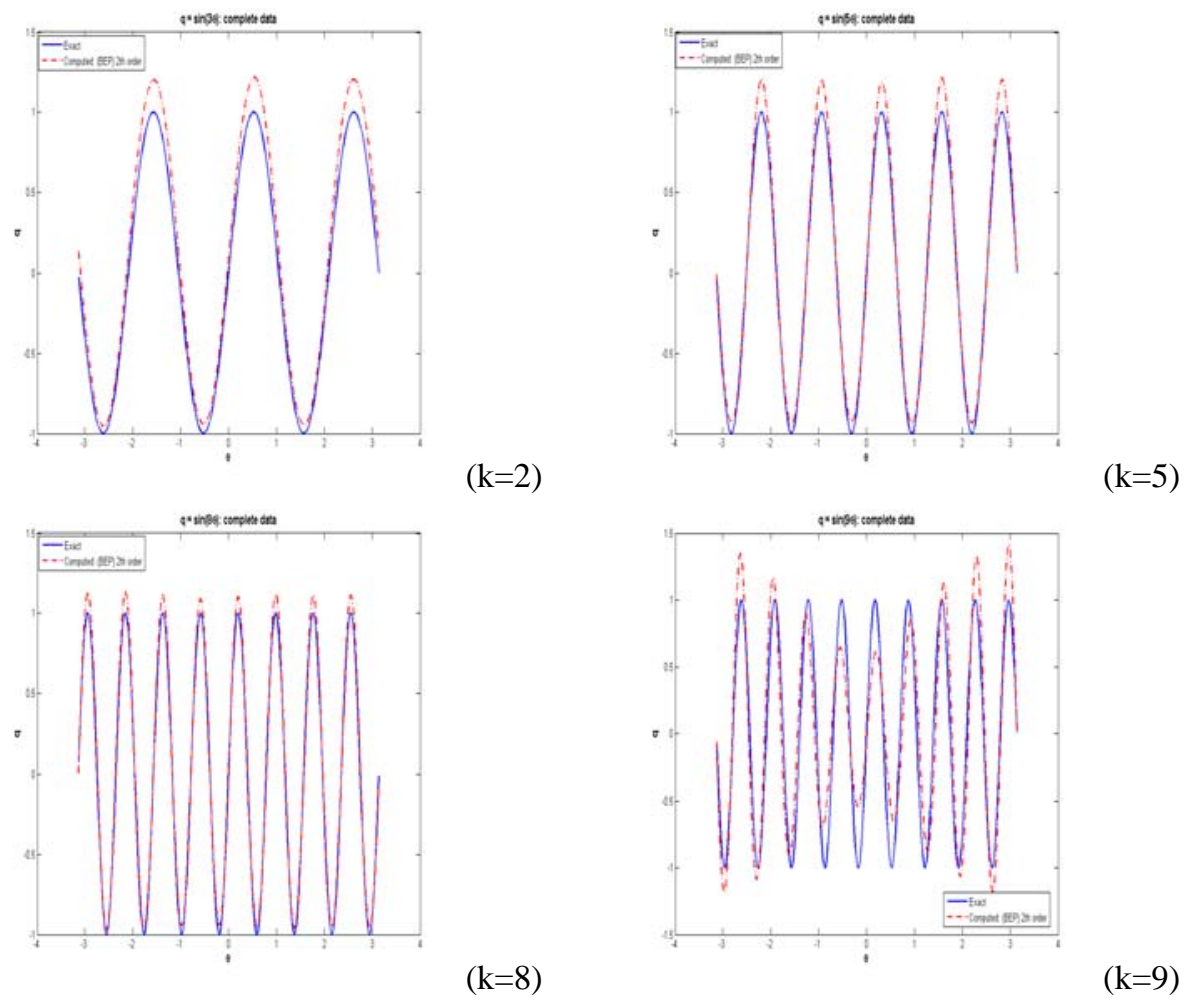

Figure 3. Recovery from full external data, 2 nd order method of oscillating impedances $\sin (k \theta), k=2,5,8,9$.
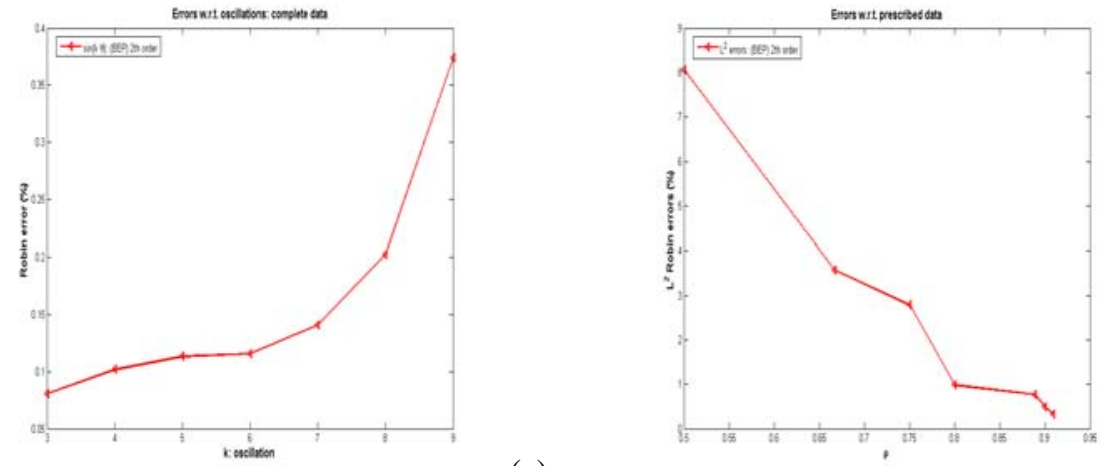

(a)

(b)

Figure 4. Errors w.r.t. number of oscillations $k$ (a) and amount of prescribed data (b) (2nd order algorithm). 
on $s \mathbb{T}$. Figure 5 shows the reconstructed impedances on $s \mathbb{T}$ with both methods presented above. As expected, the Kokn \& Vogelius method is efficient for the recovery tasks as far as the impedances do not oscillate much, whereas the (BEP) method still works for oscillatory ones.
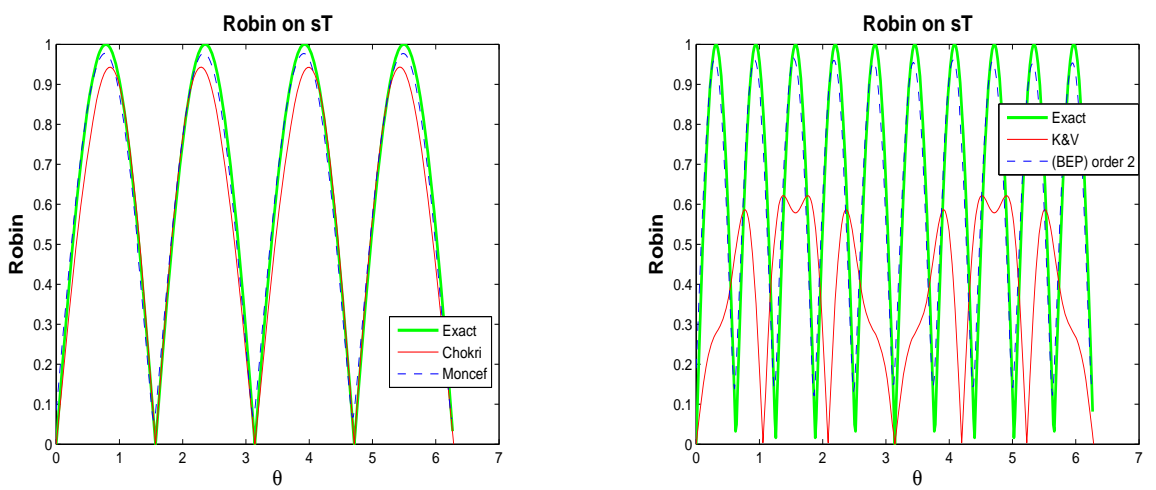

Figure 5. Recovery impedance for Kohn\& Vogelius and (BEP) methods.

\section{Conclusion}

We have described in this paper two robust numerical algorithms designed to recover the impedance in a Robin inverse problem. The first one is based on the minimization of an energy least squares function due to Kohn and Vogelius, it is an iterative process needing to solve two forward PDE problems at each iteration. The method is proved to be robust, even too much since its self-regularizing features make it uneasy to accurately capture highly oscillating impedances. As for the second, based on approximation in Hardy-Sobolev classes, its computational costs are quite low since it makes use of quasiexplicit expansions of the solutions (up to a conformal mapping). However, it is limitated to 2D situations, and to the Laplace operator too.

For both these methods, the focus has been put on the robustness issue, which is sensibility of the computed solutions with respect to noise. Noise are errors making the data violate both admissibility conditions: smoothness and compatibility. Splining the noisy data can make up for smoothness, but uncompatibility remains. What has been theoretically proved for these two algorithms is that appropriately smoothing and regularizing yields robustness.

The main issue remaining to be studied regards the search of a robustness estimate. What have been obtained so far are qualitative continuity results, with no estimates. On the numerical level, 3D experiments with the Kohn and Vogelius method would be interesting, they are indeed theoretically possible, though heavy to implement. Extending the analytic extension approach to 3D situations needs additional theory, but it would be of great interest since the method is accurate and cheap. Finally, seeking an accurate re- 
covery of oscillating impedances by methods inspired by the textures recovery in image processing [5] is another issue remaining to explore.

\section{References}

[1] Alessandrini G., Del Piero L., Rondi R., "Stable determination of corrosion by a single electrostatic measurement", in Inverse Problems, vol. 19 (2003) 973-984.

[2] AlessandRini G., SinCiCH E., "Detection of nonlinear corrosion by electrostatic measurements", in Applicable Analysis, vol. 85 (2006) 107-128.

[3] Alessandrini G., Sincich E., "Solving elliptic Cauchy problems and the identification of nonlinear corrosion", in Journal of Computational and Applied Mathematics, vol. 198 (2007) 307-320.

[4] Andrieux S., Ben Abda A., Baranger T., "Data completion via an energy error functional”, in C. R. Acad. Sci. Paris Mécanique, vol. 333 (2005) 171-177.

[5] Aubert S., Aujol A.-F., , "Modelling very oscillating signals : application to image processing”, Research Report RR-4878, INRIA, France (2003) .

[6] BARATChART L., LEBlond J., "Hardy approximation to $L^{p}$ functions on subsets of the circle avec $1 \leq p<\infty$ ", in Constructive Approximation, vol. 14 (1998) 41-56.

[7] Buttazo G., Kohn R. V., "Reinforcement by a thin layer with oscillating thickness", in App. Math. Opt., vol. 16 (1988) 247-261.

[8] ChaAbane S., Jaoua M., "Identification of Robin coefficients by the means of boundary measurements", in Inverse Problems, vol. 25 (1999) 1425-1438.

[9] Chadbane S., Elhechmi C., Jaoua M., "A stable recovery method for the Robin inverse problem”, in Mat. and Comp. in Simul., vol. 66 (2004) 367-383.

[10] ChaAbane S., Elhechmi C., Jaoua M., "Error estimates in smoothing noisy data using cubic B-Splines", in C.R. Acad. Sci. Paris, Série I, vol. 346 (2008) 107-112.

[11] Chaabane S., Fellah I., Jaoua M., Leblond J., "Logarithmic stability estimates for a Robin coefficient in 2D Laplace inverse problems", in Inverse Problems, vol. 20 (2004) 47-59.

[12] ChaAbane S., Jaoua M., Leblond J., "Parameter identification for Laplace equation and approximation in Hardy classes”, in J. Inv. Ill. P. Problems, vol. 11 (2003) 33-57.

[13] Chalendar I., Partington J.R., "Approximation problems and representations of Hardy spaces in circular domains", in Studia Math., vol. 136 (1999) 255-269.

[14] Choulli M., "An inverse problem in corrosion detection: stability estimates", in J. Inverse Ill-Posed Probl., vol. 12 (2004) 349-367.

[15] Cimetière A., Delvare F., Jaoua M., Pons F., "Solution of the Cauchy problem using iterated Tikhonov regularization", in Inverse Problems, vol. 17 (2001) 553-570.

[16] DUREn P.L., "Theory of $H^{p}$ spaces", Dover Publications, 2000.

[17] Elhechmi C., "Etude de quelques algorithmes d'identification d'un coefficient de corrosion”, Ph.D. Thesis, LAMSIN-ENIT, Tunis, (2008).

[18] ENGL H., "Necessary and sufficient conditions for convergence of regularization methods for solving linear operator equations of the first kind", in Numer. Funct. Anal. Optim., vol. 3 (1981) 201-222.

[19] FASINO D., INGLESE G., "Stability of the solutions of an inverse problem for Laplace's equation in a thin strip", in Numer. Funct. Anal. and Optimiz., vol. 22 (2001) 549-560.

[20] Inglese G., "An inverse problem in corrosion detection”, in Inverse Problems, vol. 13 (1997) 977-994. 
[21] Jaoua M., Leblond J., Mahjoub M., Partington J.R., "Robust numerical algorithms based on analytic approximation for the solution of inverse problems in annular domains", to be published in IMA J. of Appl. Math.

[22] Kabanikhin S.I., KARCheVsky A.L., "Optimizational method for solving the Cauchy problem for an elliptic equation", in J. Inverse Ill-posed Probl., vol. 3 (1995) 21-26.

[23] Kaup P., SAntosa F., "Nondestructive evaluation of corrosion damage using electrostatic measurements", in J. Nondestrutive Eval., vol. 14 (1995) 127-136.

[24] Klibanov M.V., Santosa F., "A computational quasi-reversibility method for Cauchy problems for Laplace's equation”, in SIAM J. Appl. Math., vol. 51 (1995) 1653-1675.

[25] Kohn R., Vogelius M., "Determining conductivity by boundary measurements, interior results", in Comm. Pure Appl. Math, vol. 38 (1985) 644-667.

[26] Kohn R., Vogelius M., "Relaxation of a variational method for impedance computed tomography", in Comm. Pure Appl. Math, vol. 40 (1987) 745-777.

[27] Kozlov V., MaZ’ya V., Fomin A., “An iterative method for solving the Cauchy problem for elliptic equations”, in Comput. Math. Phys., vol. 31 (1991) 45-52.

[28] Leblond J., Mahjoub M., Partington J.R., "Analytic extensions and Cauchy-type inverse problems on annular domains: stability results", in J. Inv. Ill. P. Problems, vol. 16 (2006) 189-204.

[29] Louati K., "Modèles mathématiques pour l'inspection non destructive des pipelines", Ph.D. Thesis, Ecole Polytechnique, Paris (2006).

[30] Mahjoub M., "Approximation harmonique dans une couronne et applications à la résolution numérique de quelques problèmes inverses", Ph.D. Thesis, LAMSIN-ENIT, Tunis (2008).

[31] Rudin W., "Analytic functions of class $H_{p}$ ”, in Trans. Amer. Math. Soc., vol. 78, (1955), 46-66.

[32] Santosa F., Vogelius M., XU J-R., “An effective nonlinear boundary condition for a corroding surface. identification of the damage based on steady state electric data", in Zeitschrift fur Angewandte Mathematik und Physik, vol. 49 (1998) 656-679.

[33] Sinsich E., "Lipschitz stability for the inverse Robin problem", in Inverse Problems, vol. 23 (2007) 1311-1326.

[34] SlodičKa M., VAN KeER R., "Determination of the convective transfer coefficient in elliptic problems from a nonstandard boundary condition", in J. Maryška, M. Tuma, J. Šembera, (Eds), Simulation, modelling, and numerical analysis, SIMONA 2000, 13-20, Technical University of Liberec, ISBN: 80-7083-451-X.

[35] SlOdičKa M., VAN KeER R., "A numerical approach for the determination of a missing boundary data in elliptic problems", in Appl. Math. and Comp., vol. 147 (2004) 569-580.

[36] Vogelius M., XU J.M, “A non linear elliptic boundary value problem related to corrosion modeling”, in Quarterly Applied Maths, vol. 56 (1998) 479-505. 